\title{
High Grade Astrocytic Tumor
}

National Cancer Institute

\section{Source}

National Cancer Institute. High Grade Astrocytic Tumor. NCI Thesaurus. Code C102897.

An anaplastic astrocytoma (grade III astrocytic tumor) or glioblastoma (grade IV astrocytic tumor). 